\title{
Revision of Loboederus Guérin-Méneville (Coleoptera, Elateridae, Elaterinae, Ampedini)
}

\author{
Sônia A. Casari ${ }^{1,2}$
}

\begin{abstract}
${ }^{1}$ Museu de Zoologia, Universidade de São Paulo. Caixa Postal 42494, 04218-970 São Paulo-SP, Brazil. E-mail:casari@usp.br ${ }^{2} \mathrm{CNPq}$ fellowship.
\end{abstract}

\begin{abstract}
Resumo. Revisão de Loboederus Guérin-Méneville (Coleoptera, Elateridae, Elaterinae, Ampedini). O gênero Loboederus Guérin-Méneville, 1831 incluía três espécies: L. appendiculatus (Perty, 1830), L. fleutiauxi Lesne, 1940 e L. luederwaldti Costa-Andrade, 1935. L. fleutiauxi é aqui considerada como sinônimo júnior de L. luederwaldti. A caracterização do gênero e a análise morfológica e a redescrição das duas espécies são apresentadas. O lectótipo e os paralectótipos de $L$. luederwaldti são designados e a distribuição geográfica de ambas as espécies é ampliada.

Palavras-Chave. Dicrepidiina; caracterização genérica; lectótipo; Loboederus; revisão.

Aвstract. The genus Loboederus Guérin-Méneville, 1831 formerly included three species: L. appendiculatus (Perty, 1830), L. fleutiauxi Lesne, 1940 and L. luederwaldti Costa-Andrade, 1935. L. fleutiauxi is considered as a junior synonym of $L$. luederwaldti. The generic characterization and the morphological analysis and redescriptions of both species are presented. The lectotype and paralectotypes of $L$. luederwaldti are designated and the geographical distribution of both species is widened.
\end{abstract}

Keywords. Dicrepidiina; generic characterization; lectotype; Loboederus; revision.

Loboederus Guérin-Méneville, 1831 is easily recognized by pronotum short in relation to elytra length, with hind angles highly modified, short and rounded, bearing near each lateral margin, a spatula-like prominent appendix that fits in a groove at anterior angles of elytra. Up to now, it included three neotropical species and neither the genus nor its species were studied since the original descriptions. The main purpose of this paper is to provide a taxonomic re-evaluation of these species and a carefully morfological analysis.

The material studied belongs to the following institutions (curator parenthesized): DZUP - Departamento de Zoologia, Universidade Federal do Paraná, Curitiba, Brazil (G. RosadoNeto); IBSP - Instituto Biológico, São Paulo, Brazil (S. Ide); MCNZ - Museu de Ciências Naturais, Fundação Zoobotânica do Rio Grande do Sul, Porto Alegre, Brazil (M.H.M. Galileo); MNHN - Muséum Natural d'Histoire Naturelle, Paris, France (C. Girard); MZSP - Museu de Zoologia, Universidade de São Paulo, São Paulo, Brazil (S.A.Casari).

Perty (1830) described Elater appendiculatus, from São Paulo, Brazil, characterized by antennae serrate with last antennomere "lanceolate" and hind angles of pronotum with internal appendices.

Guérin-Méneville (1831) erected Loboederus to L. monilicornis Guérin-Méneville, 1831 from São Paulo, Brazil. $\mathrm{He}$ characterized the antennae as moniliform with last article small, ovoid.

LATREILle (1834) redescribed Loboederus, only with $L$. monilicornis, considering the last antennomere with "false article" and, erroneously, recorded it from "Java". Herein, this species is understood as being L. luederwaldti Costa-Andrade, 1935, described a century later.
CASTelnau (1840) redescribed the genus and considered E. appendiculatus Perty, 1830 as synonym of L. monilicornis Guérin-Méneville, 1831.

LACORDAIRE (1857), treating of the genus, described the antennae as filiform in females and serrate in males, with last antennomere with rounded apex. According to him, Guérin had examined only females because he did not describe the lamellae of tarsi, invisible in females, and also, had not mentioned the sexual differences of antennal shape and lenght. He considered the genus formed only by L. appendiculatus Perty, from São Paulo, Brazil, but stated that "M. GuérinMéneville, qui l'a décrit presque em même temps que M. Perty, l'a nommé L. monilicornis...". He commented the error of Latreille recording it from Java.

CANDĖze (1859) redescribed the genus and species, and following LACORDAIRE (l.c.), considering L. monilicornis as synonym of $L$. appendiculatus. He repeated the comments that these species were described almost at same time, and the Latreille's record from Java. He also stated that Latreille verified only one lamella under $3^{\text {rd }}$ tarsomere, but according to him, this fact is justified by variable size of lamella of $2^{\text {nd }}$ tarsomere, better visible at protarsi of males and almost invisible in females.

Schwarz (1906) treating of the "Tribus Dicrepidiini" presented a key to genera and redescribed the "Genus Loboderus [sic]Guérin", formed by L. appendiculatus.

Hyslop (1921) followed Castelnau (l.c.) and considered Loboederus monilicornis as type species. In fact, this species was a synonym of $L$. appendiculatus.

CAMARgo-Andrade (1935) presented a historical review of the genus discussing the previous authors and described a 
new species, $L$. luederwaldti. He also redescribed the genus and $L$. appendiculatus.

LESNE (1940) described L. fleutiauxi, herein considered as a synonym of $L$. luederwaldti.

\section{Loboederus Guérin-Méneville, 1831}

Loboederus Guérin-Méneville, 1831: 9; Latreille, 1834: 148; Castelnau, 1840: 239; Hyslop, 1921: 653; Camargo-Andrade, 1935: 438.

Lobederus [sic]; Lacordaire, 1857: 167; Candèze, 1859: 9, 78; Schenkling, 1925: 82.

Loboderus [sic]; Gemminger \& Harold, 1869: 1514 (cat.); Schwarz, 1906: 61, 69; Lesne, 1940: 35.

Lobaederus [sic]; Blackwelder, 1944: 298.

Type species: Loboederus monilicornis Guérin-Méneville, 1831 (=Elater appendiculatus Perty, 1830) by monotypy.

Pubescence (Figs. 52, 53) long and dense; frons prominent anteriorly, grooved at middle; median concavity of frons wider on distal half; nasal plate almost as wide as high. Antennae long, serrate and with 11 antennomeres; in male surpassing hind angles of pronotum by 4 antennomeres, shorter in female. Mandibles (Figs. 31-34) with small subapical tooth, longitudinal sinuous dorsal carina and moderately long laterodorsal setae; almost symmetrical, only with apex and subapical tooth shorter in left mandible; penicillus formed by band of short setae. Labrum semicircular; epipharynx (Fig. 36) densely clothed with thin yellowish setae, shorter near base; two sclerites near base and many sensories. Hypopharynx (Fig. 40) densely clothed with thin yellowish setae directed to middle; with a longitudinal median sclerite. Pronotum transverse, narrower on anterior half; hind angles short and rounded bearing, innerly each lateral margin, a spatula-like appendix outwardly directed, fitting to elytral groove. Scutellum semi-elliptical and declivous. Prosternum wide, moderately convex with punctation coarse and moderately sparse, prominent between procoxae; prosternal spine short; hypomera strongly declivous to lateral margins with punctation coarse and moderately dense, more concentrate near margins. Mesosternal cavity (Fig. 30) narrow, V-shaped with borders slightly declivous. Metacoxal plate narrowed laterally, sinuous on internal half(Fig.11). Tibial spurs present. Tarsomeres 2-3 bearing small lamellae, almost invisible in females. Elytra strongly declivous forward behind scutellum; striae marked by coarse punctures, strongly grooved near base; interstices unequal, 1, 3, 5, 7, narrower; apices conjointly rounded; marginate laterally, margin wider apicad. Wing (Fig. 10) with a wedge cell (W) and apical W-like sclerotization. Parameres of aedeagus separated basally (Figs. 13, 14). Ovipositor very long, coxites short with syli (Figs. 22, 23).

\section{Loboederus appendiculatus (Perty, 1830)}

$$
\text { (Figs. 1-23, 52) }
$$

Elater appendiculatus Perty, 1830: 21, fig. 5.

Loboederus appendiculatus; Gemminger \& Harold, 1869: 1514 (cat.); Lacordaire, 1857: 170; Candéze, 1859: 79; Schenkling, 1925: 82;
Blackwelder, 1944: 298 (cat.).

Loboederus monilicornis Guérin-Méneville, 1831: 9; Castelnau, 1840: 239; Lacordaire, 1857: 170 (syn.). Syntype: "Type; Collection A. Sallé; Lobederus monilicornis Guer. Mag. Zool., 1832 pl. 9 (type) Brésil; Collection Guérin; Collection Fleutiaux", (MNHN) (examined).

Length: 15-20 mm. Integument reddish-brown with antennae, legs and appendices of hind angles of pronotum darker; pubescence golden, moderately long and dense, longer on pronotum, more concentrated on lateral margins and base of hind angles (Fig.52).

Frons (Figs. 3, 4) strongly prominent anteriorly; punctation coarse, dense and heterogeneous. Antennae: antennomeres elongate, longer apicad in male (Fig. 1) and transverse or subquadrangular in female (Fig. 2); last antennomere elongate, sub-elliptical. Labrum (Fig. 5) transverse, semicircular, with very long setae, lacking only in narrow basal band. Maxillae (Fig. 8): galea and lacinia brush-like; mediostipe concave longitudinal medially with longitudinal weak carina parallel external margin; band of long setae between carina and lateral margin and short setae near middle; basistipe subtriangular with moderately long setae, some of them longer; cardo with few short setae; palpi setous, distal palpomere slightly securiform. Labium (Fig. 7): prementum membranous, ligula bilobed, marginate by short yellowish setae; setae moderately long between palpigerous apices; palpigerous separate, bearing moderately long setae; palpi setous; distal palpomere slightly securiform; mentum transverse, translucent in narrow anterior band, bearing many setae moderately long on basal half; submentum fused to gula, transverse, clothed with setae of varied sizes. Epipharynx, mandibles and hypopharynx like $L$. luederwaldti.

Pronotum (Fig. 4) strongly narrowed on anterior half; lateral margins parallel on basal half; strongly convex, slightly grooved longitudinal medially and parallel lateral margins; anterior margin sinuous, prominent at middle; dense and coarsely punctate; appendices of hind angles moderately punctate and fitting to elytra (Fig. 6); posterior margin downwards and notched at middle; median basal tubercle flat and rounded. Apex of prosternal spine (Fig. 9) narrowed apically and compressed laterally. Prosternal lobe downwardly directed, coarse and densely punctate. Pronotosternal sutures (Fig. 9) curved, strongly grooved in anterior half and forming a channel. Tarsus (Fig. 12). Wings (Fig. 10). Elytra narrowed in distal third with apices conjointly rounded (Fig. 52).

Male. Tergite 8 (Fig. 18) elongate, gradually narrowed to apex; distal margin rounded; translucent in narrow basal band, clothed with short setae, longer at margins. Sternite 8 (Fig. 15) transverse, slightly narrowed apicad, distal margin strongly notched at middle; translucent in a lateral band and at middle; setae moderately long near lateral margins and at middle. Sternite 9 (Fig. 16) elongate, sub-elliptical, strongly narrowed in distal half; setae moderately long at distal fourth. Tergite 9 (Fig. 17) transverse, basal margin strongly and distal slightly notched; densely punctate laterally; tergite 10 longer than wide and fitted at apex of tergite 9, gradually narrowed apicad, 

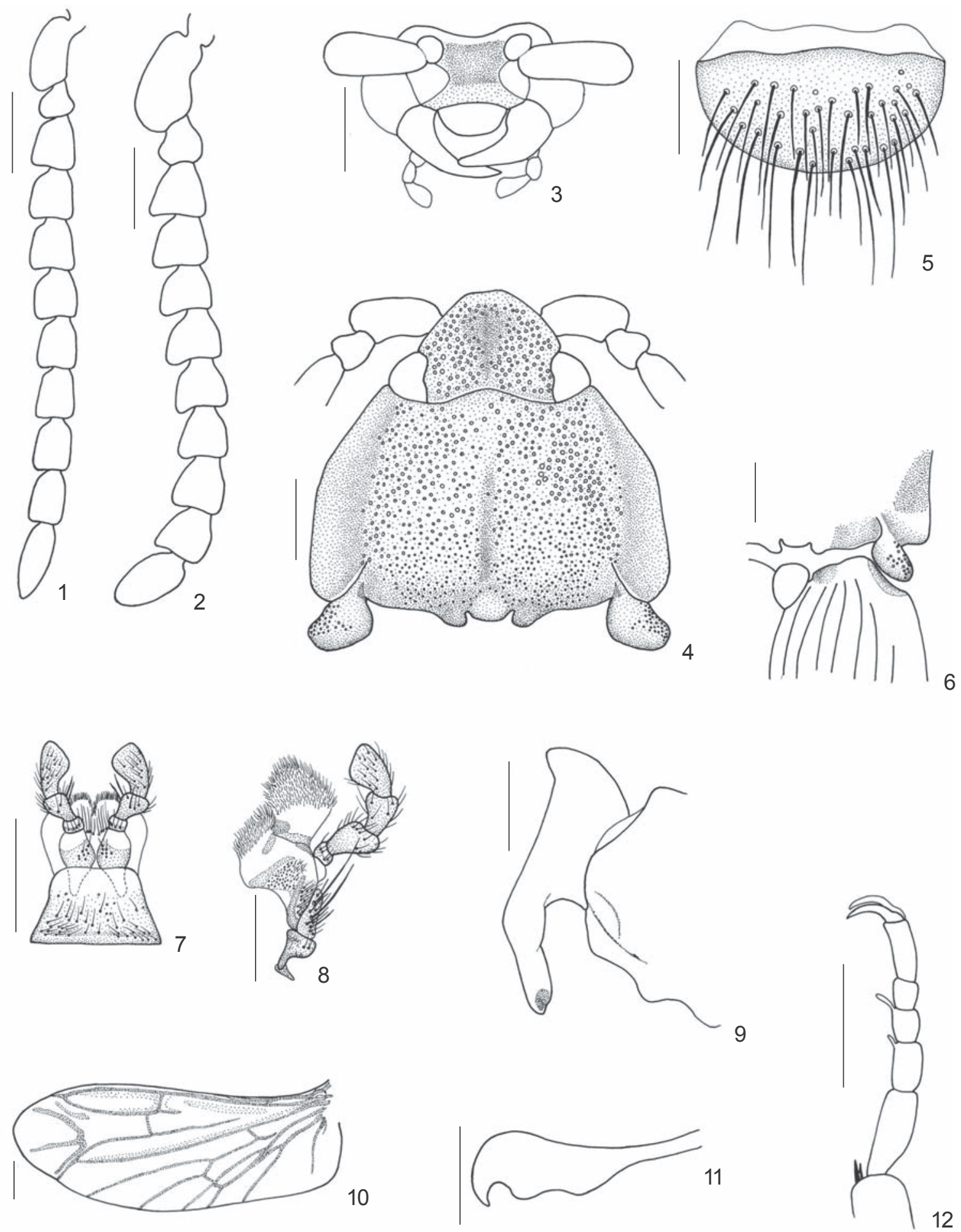

Figs. 1-12. Loboederus appendiculatus (Perty, 1830). 1-2 antennna (male, female); 3, head showing nasal; 4, head and pronotum; 5, labrum; 6, basal margins of pronotum and elytra (lateral); 7, labium; 8, maxilla; 9, prosternum and pars of hypomera (lateral); 10, wing; 11, metacoxal plate; 12, protarsus; bars $=1 \mathrm{~mm}$, except figs. $5-8=0.5 \mathrm{~mm}$.

punctate laterally and bearing short setae; apex with fringe of short setae. Aedeagus (Figs. 13, 14): basal third of parameres wider and narrowed basad; each paramere with subapical lateral notch forming one, not prominent, lateral tooth on distal third; moderately long setae dorsal and ventral; median lobe longer than parameres, narrowed near middle and widened at distal half, with apex constricted.

Female. Tergite 8 (Fig. 19) slightly wider than long, 

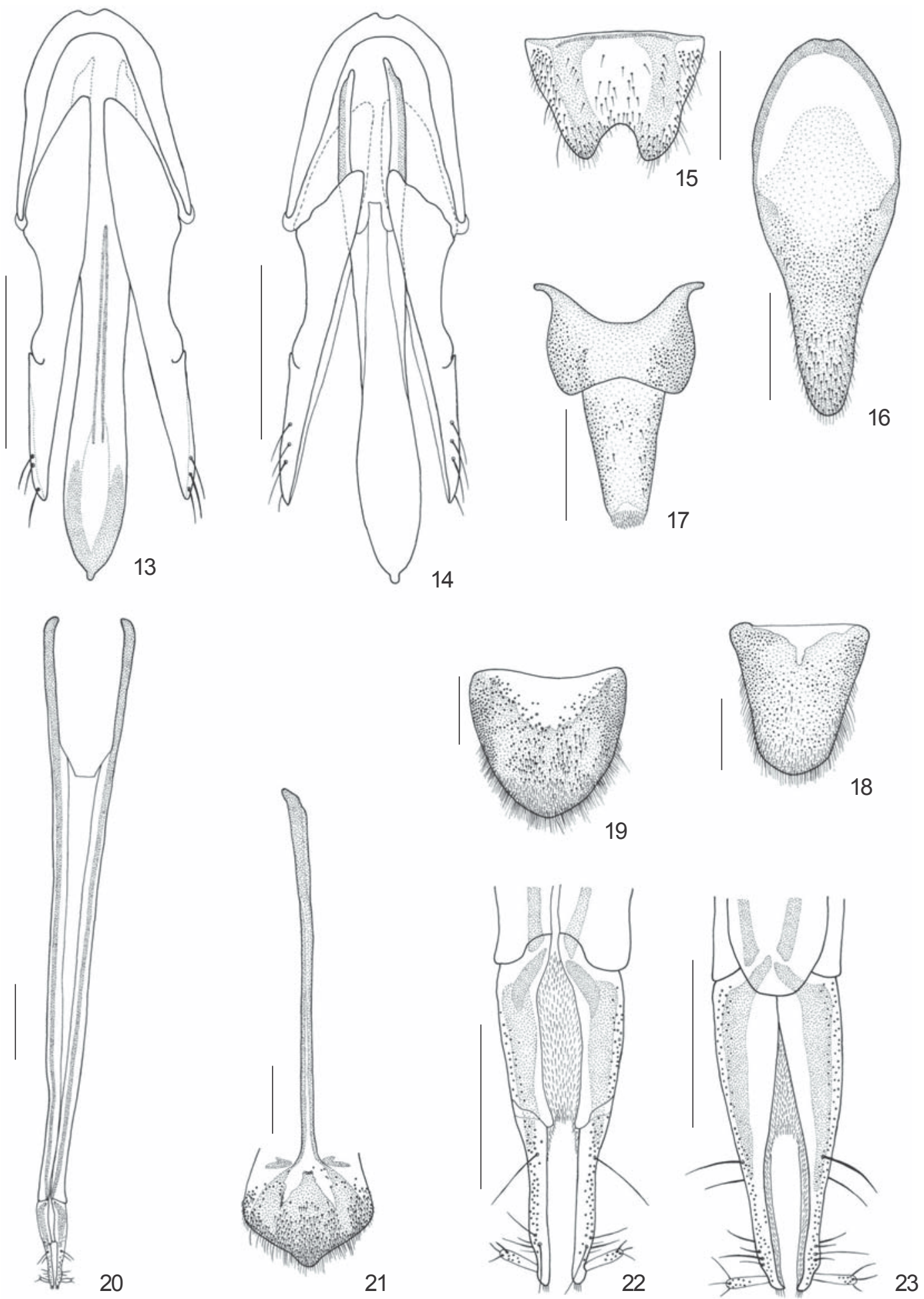

Figs. 13-23. Loboederus appendiculatus (Perty, 1830). Male: 13-14, aedeagus (ventral, dorsal); 15, sternite 8; 16, sternite 9; 17, tergites 9-10; 18, tergite 8 . Female: 19, tergite 8 ; 20, ovipositor; 21, sternite $8 ; \mathbf{2 2 - 2 3}$, coxites (ventral, dorsal); bars $=1 \mathrm{~mm}$, except figs. $22,23=0.5 \mathrm{~mm}$.

narrowed apicad; lateral margins rounded; translucent in basal subtriangular band; clothed with short setae, longer near margins. Sternite 8 (Fig. 21) subpentagonal; distal margin prominent at middle; translucent in 4 ray-like areas starting at base; setae moderately long on distal half, longer near margins; basal sclerite 3.43 times sternite length. Ovipositor (Figs 20, $22,23)$ long; coxites short, ventrally apparently 2 -segmented, with laterodistal setous stylus near apex; baculli 6.6 times sternite length. 
Remarks. Up to now, L. appendiculatus was recorded only from São Paulo and Minas Gerais. Based on the material examined, with new records from northern of Brazil, the geographical distribution is widened: Brazil (Goiás, Distrito Federal, Bahia, Mato Grosso, Mato Grosso do Sul, Minas Gerais, São Paulo, Paraná).

Material examined. BRAZIL. 1 ex. (MNHN). Goiás: Anápolis (Chácara Tangará), 1 ex. (MZSP); Leopoldo Bulhões, 1 ex. (IBSP); Rio Verde, 1 ex. (MZSP). Distrito Federal: Brasília (Cidade Universitária), 1 ex. (MZSP). Bahia: Encruzilhada, 960 m, 2 exs. (DZUP). Mato Grosso: Chavantina, $1 \mathrm{M}$ (dissected) (MZSP); Dourados, 1 ex. (DZUP); Rio Brilhante, 1 ex. (DZUP); Salobra (Instituto Oswaldo Cruz, Zona da N. O. B.), 1 ex. (MZSP); Utiariti (Rio Papagaio), 1 ex. (MZSP). Mato Grosso do Sul: Fátima do Sul (Sítio Alpha), 1 ex. (MZSP). Minas Gerais: Arinos, 1 ex. (MZSP); Uberaba, 3 exs (MNHN);Uberlândia, 1 ex. (MZSP). São Paulo: Andes, 3 exs (MZSP); Batatais, 1 ex. (MZSP); Botucatu, 5 exs. (1 F dissected) (MZSP); Itápolis, 1 ex. (MZSP); Itu, 1 ex. (MZSP); Nova Europa (Faz. Itaquerê), 2 exs. (MZSP); Piraju, 2 exs. (MZSP); Pirassununga, 2 exs. (MZSP); Regente Feijó, 1 ex. (MZSP); Ribeirão Preto (Tamanduá), 2 exs (MZSP); (Coqueiros), 1 ex. (MZSP); (Fac. Medicina), 1 ex. (MZSP); Rio Claro (Campus), 1 ex. (MZSP); São José dos Campos, 1 ex. (MZSP); São Paulo (Cantareira), 1 ex. (MZSP). Paraná: Campo Mourão, 1 ex. (DZUP). Localities recorded by literature: Minas Gerais: Lagoa Santa. São Paulo: Franca, Leme, São Paulo.

\section{Loboederus luederwaldti Camargo-Andrade, 1935}

$$
\text { (Figs. 24-51, 53) }
$$

Loboederus luederwaldti Camargo-Andrade, 1935: 441; Blackwelder, 1944: 298 (cat.)

Loboederus monilicornis Latreille, 1834, non Guérin-Méneville, 1831. Loboederus fleutiauxi Lesne, 1940: 35. Syntype: "S. Paole, Brasil; Museum Paris coll. E. Fleutiaux; Type, Loboderus fleutiauxi Lesne P. Lesne vid.; Type", (MNHN) (examined). Syn. nov.

Length: 18-24 mm. Integument reddish-brown; pubescence long and dense, yellowish-white, longer on pronotum, denser near lateral margins and hind angles of pronotum and lateral margins of elytra; pubescence lacking on elytral striae giving to elytra striped appearance (Fig. 53).

Frons (Figs. 26, 29) moderately prominent anteriorly, narrowed lateroapically and near base; punctation coarse and dense. Antennae: antennomeres elongate apicad in male (Fig. 24) and subquadrangular in female (Fig. 25); last antennomere constricted near apex. Labrum (Fig. 35) slightly wider than long, distal margin prominent and rounded, long setae on distal half. Epipharynx (Fig. 36). Mandibles (Figs. 31-34). Maxilla (fig. 37): galea and lacinia brush-like; mediostipe with longitudinal carina, parallel external margin, forming a concave setous area external carina; short setae near base of galea; basistipe subtriangular with long setae; cardo with moderately long setae; palpomere distal slightly securiform. Labium (Fig. 39): prementum membranous; apex of ligula marginate by short yellowish setae; setae moderately long at middle, near distal margin; palpigerous partially sclerotized, separate, bearing moderately long setae; distal palpomere slightly securiform; mentum transverse, translucent in a narrow anterior band, bearing many setae moderately long on basal half, two of them longer; submentum fused to gula, transverse, clothed with setae of varied sizes. Hypopharynx (Fig. 40).

Pronotum (Fig. 26) wider than long, narrowed on proximal third; strongly convex near posterior margin, convexity decreasing anteriad; strongly grooved longitudinal medially and parallel lateral margins on basal 2/3; anterior margin sinuous; lateral margins raised on basal $2 / 3$; posterior margin downwards and notched at middle; median basal tubercle flat and rounded; hind angles rounded with inner prominent appendix, densely punctate, fitted at elytra (Fig. 27); punctation coarse, very dense and heterogeneous. Prosternal spine (Fig.28) short, with apex rounded and compressed laterally; prosternal lobe downwardly directed, coarse and densely punctate. Pronotosternal sutures curve, grooved on anterior half. Elytra with apices conjointly rounded, in some specimens with very tiny sutural spine.

Male. Tergite 8 (Fig. 43) slightly longer than wide, wider near base, slightly narrowed apicad; clothed with short setae (represented by punctures on figure), margin with a fringe of long setae. Sternite 8 (Fig. 45) tranverse, subrectangular, slightly narrowed to apex; fore angles rounded; anterior margin slightly notched at middle; clothed with moderately long setae, more concentrated near margins. Sternite 9 (Fig. 46) elongate, narrowed on distal half; densely punctate and with moderately long setae on distal third; distal margin, slightly notched at middle. Tergite 9 (Fig. 44) wider than long, slightly narrowed apicad; strongly notched at basal and distal margins; densely punctate near lateral and anterior margins; a few moderately short setae near anterior angles; tergite 10 fitted at apex of tergite 9 , elongate, constricted at apex; densely punctate, apex membranous and clothed with short setae. Aedeagus (Figs. 41, 42): parameres with sides almost parallel, separate basally, narrowed laterally near middle and near base; apex constricted; lateral margin notched near middle forming a tooth backwardly directed; moderately long setae near apex; median lobe longer than parameres, widened near apex; apices constricted.

Female. Tergite 8 (Fig. 49) slightly longer than wide, wider basally, narrowed apicad; anterior margin rounded; translucent in a large triangular basal area; densely punctuate and marginate by moderately long setae. Sternite 8 (Fig. 51) semi-elliptical, translucent in small lateral and median bands, densely clothed with moderately short setae; basal sclerite 3.8 times sternite length. Genitalia: coxites short (Figs. 47, 48, 50), ventrally aparently 2 -segmented with laterodistal stylus; densely punctate laterally; setous innerly at distal half; baculli very long, 8.6 times coxites length.

Remarks. Latreille (1834) redescribed L. monilicornis Guérin-Méneville based on material from a different species, that presented the last antennomere with apex constricted. Based on his redescription it is here concluded that this material belongs to L. luederwaldti Camargo-Andrade,1935, described a century later.

Loboederus luederwaldti Camargo-Andrade (1935) was described based on 35 specimens, males and females, all from São Paulo, Capital, without the holotype and paratype 

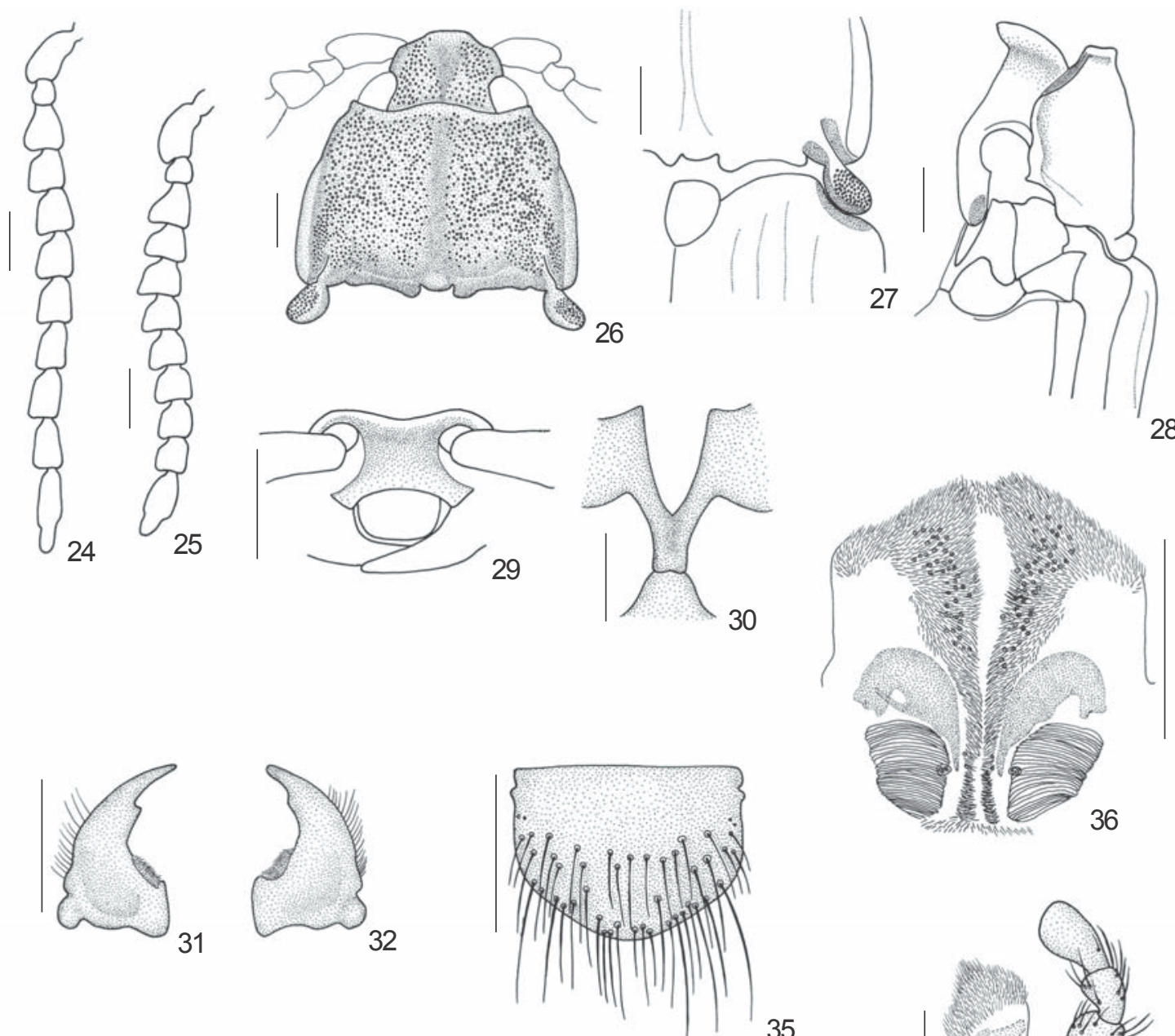

28
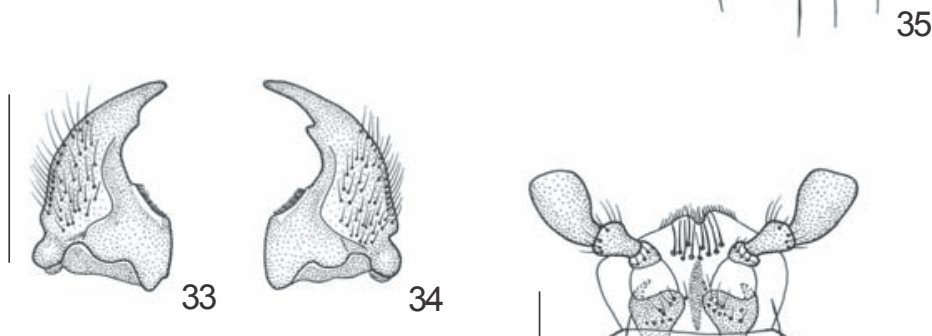

35

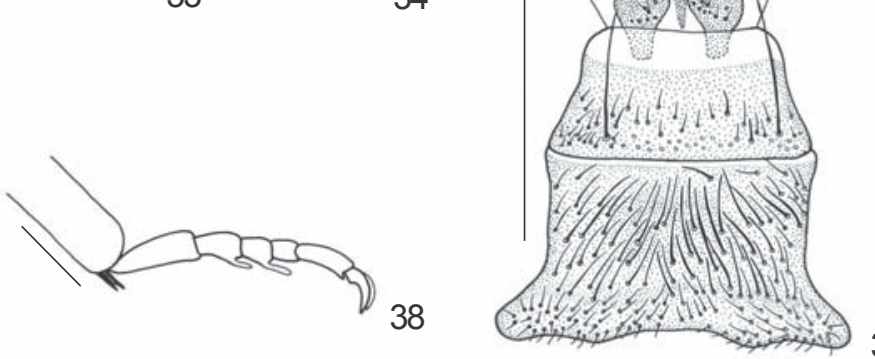

39

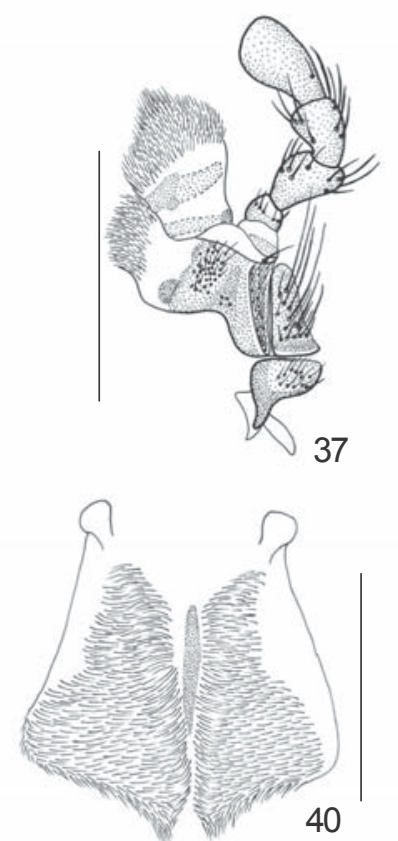

Figs. 24-40. Loboederus luederwaldti Camargo-Andrade, 1935. 24-25, antenna (male, female); 26, head and pronotum; 27, basal margins of pronotum and elytra (lateral); 28, prothorax and mesothorax (lateral); 29, head showing nasal; 30, mesosternal cavity; 31, 34, right mandible (ventral, dorsal); 32, 33, left mandible (dorsal, ventral); 35, labrum; 36, epipharynx; 37, maxilla; 38, protarsus; 39, labium; 40, hypopharynx; bars $=1 \mathrm{~mm}$, except figs. $35,36,40=0.5 \mathrm{~mm}$.

designations. I examined nine specimens of this species, from MZSP, labelled as "Cotipo", some of them from cities near São Paulo. Lectotype and paralectotype designations, as follows. Lectotype: “Cotipo; São Paulo, Ypiranga, III-29, R. Spitz leg.;
Loboederus luederwaldti Camargo, 1935" (MZSP).

Paralectotypes: "Cotipo; São Paulo, Alto da Serra, III-24, R.

Spitz leg.; Anoplischius longicornis Blach.; Fleutiaux det.; Loboederus luederwaldti Camargo, 1935”(MZSP); “Cotipo; 

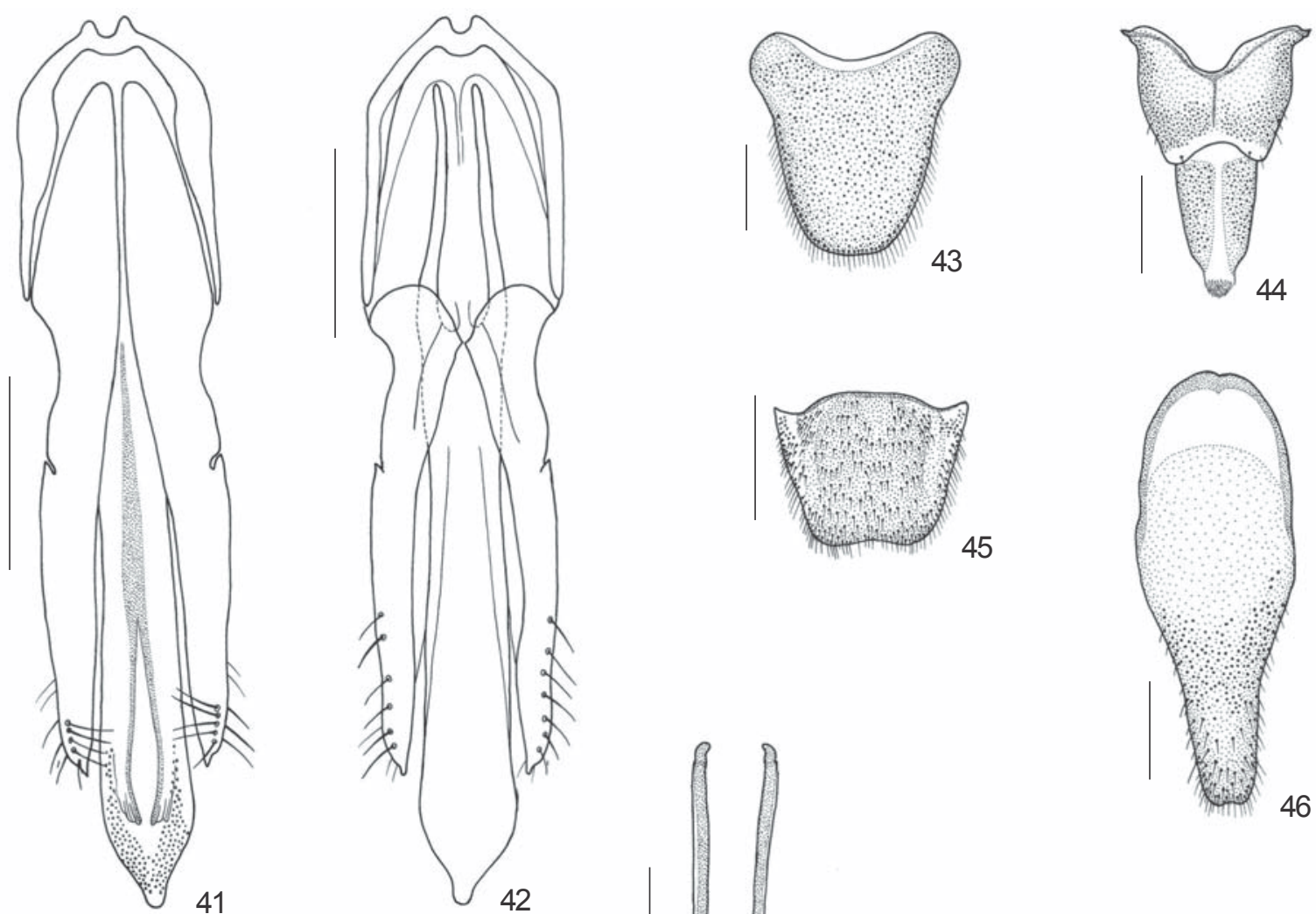

41
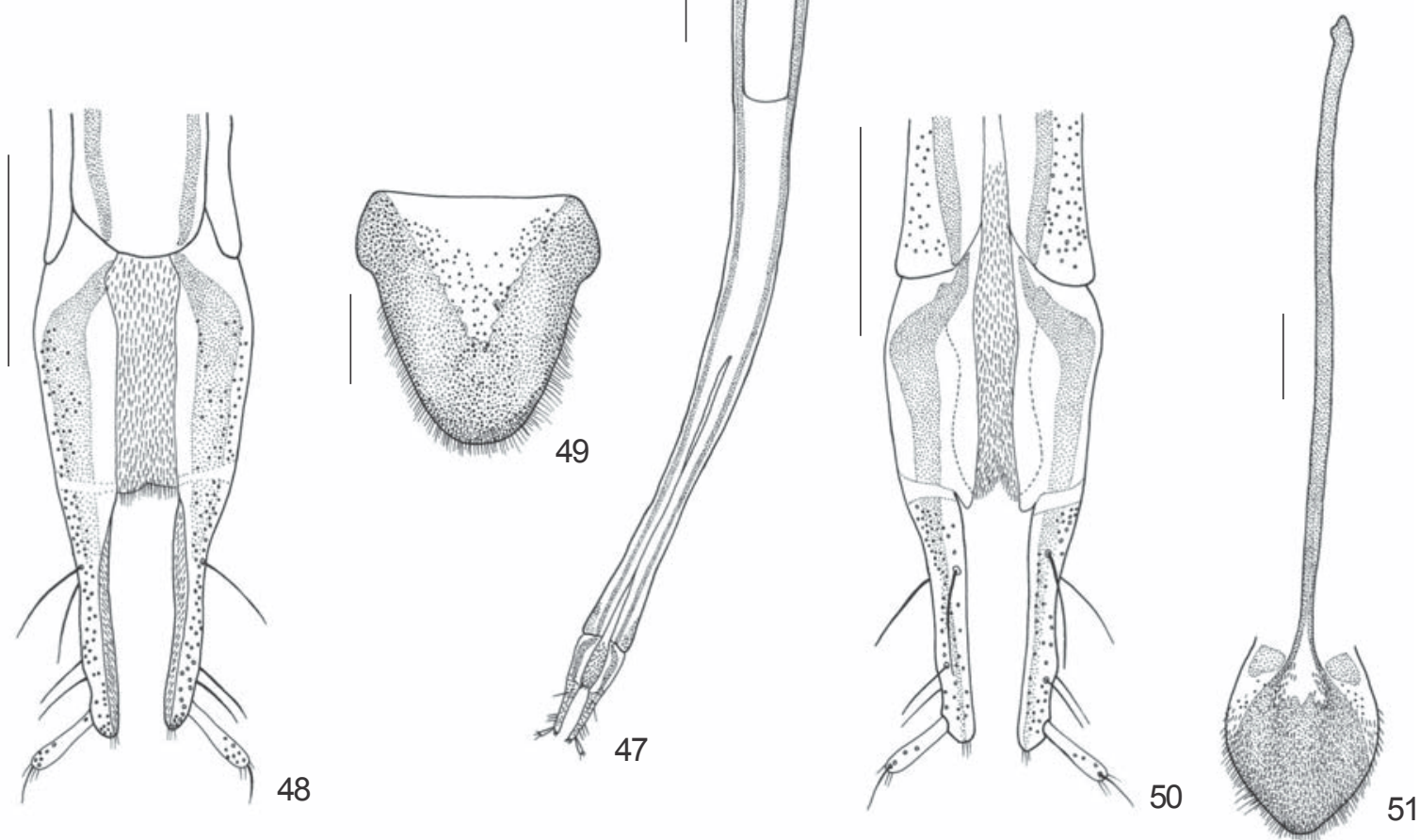

Figs. 41-51. Loboederus luederwaldti Camargo-Andrade, 1935. Male: 41-42, aedeagus (ventral, dorsal); 43, tergite 8; 44, tergites 9, 10; 45, sternite $8 ; \mathbf{4 6}$, sternite 9 . Female: $\mathbf{4 7}$, ovipositor; 48, 50, coxites (dorsal, ventral); $\mathbf{4 9}$, tergite 8 ; $\mathbf{5 1}$, sternite 8 ; bars $=1 \mathrm{~mm}$, except figs. 48,50 $=0.5 \mathrm{~mm}$.

São Paulo, São Bernardo, III-27, R. Spitz leg.; L 154; Loboederus luederwaldti Camargo, 1935”(MZSP); “Cotipo; São Paulo, São Bernardo, 1-X-28, R. Spitz leg.; L 154; Loboederus luederwaldti Camargo, 1935"(MZSP); same data, plus "Anoplischius longicornis Blanch., Fleutiaux det. 34; Loboederus luederwaldti Camargo, 1935” (MZSP); “Cotipo; São Paulo, Ypiranga, II-27, Spitz leg.; Loboederus luederwaldti Camargo, 1935” (MZSP); “Cotipo; São Paulo, Ypiranga, 19525; Loboederus luederwaldti 

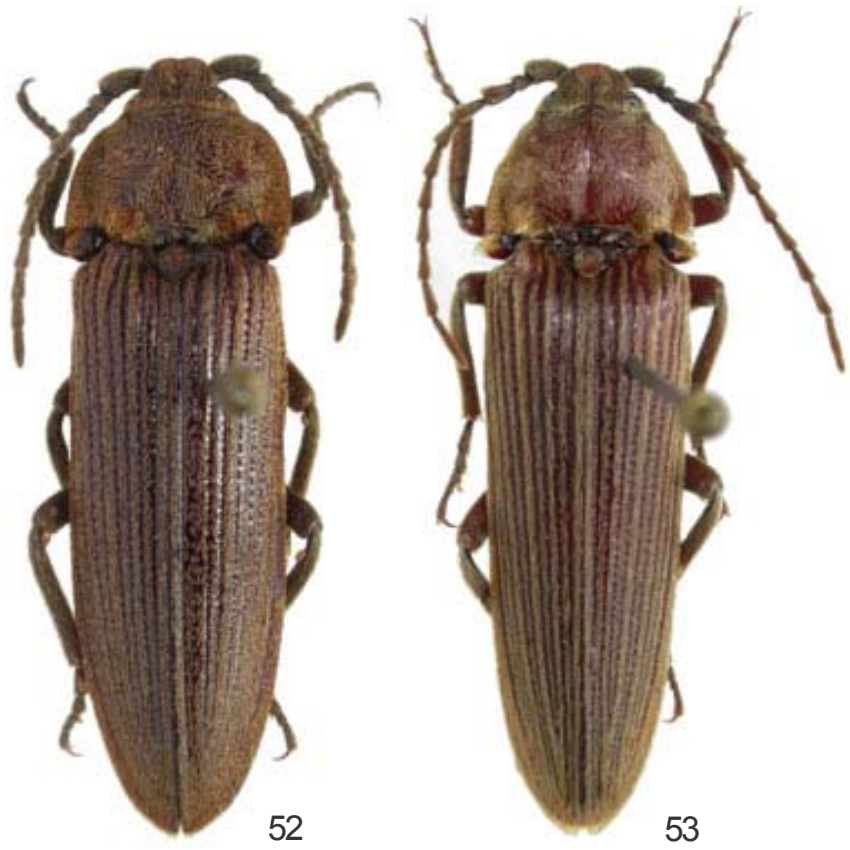

Figs. 52-53. Habitus. 52, Loboederus appendiculatus; 53, Loboederus luederwaldti. Length, respectively, 17, $20 \mathrm{~mm}$.

Camargo, 1935”(IBSP); “Cotipo; Ypiranga, E. S. Paulo; L. 152; Loboederus luederwaldti Camargo, 1935”(MZSP); "Cotipo; 22009; Loboederus luederwaldti Camargo, 1935”(IBSP).

LESNE (1940) described Loboderus [sic] fleutiauxi, and presented a discussion on the morphology of the genus, especially the conformation of hind angles of pronotum. He did not mention about the antennal shape of last antennomere. According to him it is probably that the members of this genus have myrmecophilous or termitophilous habits. Comparing the type of this species with the material of $L$. luederwaldti it was concluded that they are synonyms.

A comparison of $L$. appendiculatus with $L$. luederwaldti shows the following differences (the latter parenthesized): 1) pubescence longer in $L$. luederwaldti; 2) frons stronger prominent in L. appendiculatus; 3) last antennomere elongate, sub-elliptical (constricted near apex); 4) labrum transverse, band-like (slightly wider than long); 5) setae near longitudinal carina of mediostipe longer (shorter and denser); 6) pronotum slightly grooved longitudinal-medially (strongly grooved longitudinal-medially); 7) appendix of hind angles of pronotum nearer lateral margin and punctation of pronotum and apendices denser in L. luederwaldti; 8) elytral striae more coarsely punctate in L. appendiculatus; 9) distal margin of sternite 8 of male stronger notched in L. appendiculatus; 10 ) sternite 9 of male elliptical with distal margin rounded (narrowed at distal half with apex slightly notched); 11) parameres notched laterally near distal third (notched laterally near middle); 12) sternite 8 of female subpentagonal (elliptical).

Based on the material examined, the geographical distribution is wide spread, especially to northern Brazil and to Uruguay.

Material examined. BRAZIL. Amazonas: São Jerônimo, 1 ex (MCNZ). Pará: Jacareacanga, 23 exs. (DZUP), 1 ex. (MZSP). Mato Grosso. Corumbá, Loboderus fleutiauxi Lesne, Cotype, Coll. Fleutiaux, 1 ex. (MNHN); Rosário-Oeste, 1 ex. (MZSP). Minas Gerais: Rio Verde, 400 m, 3 exs. (DZUP). Rio de Janeiro: Itatiaia, 1 ex. (MZSP); Nova Friburgo, 1 ex. (MNHN). São Paulo: 2 exs. (MNHN); Alto da Serra, 1 Paralectotype (MZSP); Anhembi (Barreiro Rico), 1 ex. (MZSP); (Fazenda Barreiro Rico), 2 exs. (MZSP); Barueri, 5 exs. (MZSP); (estômago de Bufo ictenicus), 1 ex. (MZSP); Botucatu, 1 ex. (MZSP); Itu, 1 ex. (MZSP); (Fazenda Pau d'Alho) 1 ex. (MZSP); Jarinú, 1 ex. (MZSP); Piracicaba, 1 ex. (MZSP); São Bernardo, 3 Paralectotypes (MZSP); São Paulo, (Jabaquara), 2 exs (MZSP); (Santo Amaro), 1 ex. (IBSP); (Sumaré), 1 ex. (MZSP); (Ypiranga), Lectotype, 2 Paralectotypes (MZSP), 1 Paralectotype (IBSP). URUGUAY. Rivera, Sierra de la Aurora, Arroyo de la Aurora, 3 exs. (MZSP). Locality not stated: 22009; 1 Paralectotype (IBSP). Locality recorded by literature: Mato Grosso: Corumbá.

Acknowledgement. To Guilherme Ide Marques dos Santos (MZSP) by taking the pictures.

\section{REFERENCES}

Blackwelder, R. E. 1944. Checklist of Coleopterous insects of Mexico, Central America, the West Indies and South America. Bulletin of the United States National Museum 185(2): 280-303.

Camargo-Andrade, C. A. 1935. O Genero Loboederus. Revista do Museu Paulista 19: 431-442, figs.

CandĖze, E. 1859. Monographie des Élatérides. Mémoires de la Societé Royale de Sciences de Liège 14: 1-543, 7 pls.

Castelnau, F. L. N. C. Laporte Comte DE. 1840. Histoire naturelle des animaux articulés. Paris, v. 1, $324 \mathrm{p}$.

Gemminger, M. \& E. Von Harold. 1869. Catalogus Coleopterorum hucusque descriptorum synonymicus et systematics, Monachii, v. 5, p. 1347-1808.

GuÉRIN-MÉnevilLe, F. E. 1831. [Description of Loboederus monilicornis]. Magasin de Zoologie 1(1): 9.

Hyslop, J. A. 1921. Genotypes of the elaterids of the world. Proceedings of the United States National Museum 58: 621-680.

Lacordaire, J. T. 1857. Genera des Colèoptères... Paris, Librairie Encyclopédique de Roret, v.4, 579 p.

Latreille, P. A. 1834. Distribution méthodique et naturelle des genres de diverses tribus d'insectes coléoptères, de la famille des serricornes. Annales de la Societé Entomologique de France 3: 113-170.

Lesne, P. 1940. Remarques sur divers Élatérides [Col.]. Bulletin de la Société entomologique de France 45(3): 33-40.

Perty, J. A. M. 1830. Insecta Brasiliensa. In: Delectus animalium articulatorum... (fasc. 1), pp. 1-60, illus.

Schenkling, S. 1925. Elateridae I. Pars 80. In: Coleopterorum Catalogus auspiciis et auxilio W. Junk. Berlin, W. Junk, 263 p.

Schwarz, O. C. E. 1906. Coleoptera Fam. Elateridae. Fasc. 46A p. 1112. In: P. Wrstman (ed.). Genera Insectorum. Bruxelles, P. Wystman. 